\title{
Physical exercise intervention via telerehabilitation in patients with neurological disorders: a narrative literature review
}

\author{
Fatih Özden ${ }^{1}$, Mehmet Özkeskin²*i] and Süleyman Mert Ak $^{3}$
}

\begin{abstract}
Background: In recent years, telerehabilitation applications have increased with the rapid development of mobile technology. Remote rehabilitation services have utmost importance in chronic neurological disorders. The aim of this narrative literature review was to discuss the physical exercise interventions via telerehabilitation in patients with neurological disorders. The literature search was conducted via PubMed using the neurological pathology terms in the MeSH (Medical Subject Headings) database. Physical exercise-based studies within the scope of neurological rehabilitation were included in the study. The contents of the studies were discussed with narrative synthesis.
\end{abstract}

Results: A total of 329 studies were obtained in the initial search. Twelve studies including cases of multiple sclerosis (MS), stroke, parkinson's disease, intracranial tumors, spinal-cord injury were interpreted. A vast majority of studies (50\%) was conducted with stroke cases. On the other hand, half of the studies addressed the specific results of balance or balance-falling. The results of the studies were discussed comprehensively.

Conclusion: Physical exercise with telerehabilitation provides productive results to improve quality of life, muscle strength-endurance, hand function, balance, aerobic capacity in neurologic rehabilitation.

Keywords: Neurological rehabilitation, Physical exercise, Remote rehabilitation, Telehealth, Telemedicine, Telerehabilitation

\section{Introduction}

Telerehabilitation is defined as rehabilitation delivered remotely via telephone, videoconferencing, computer software or smart device applications [1]. Many healthcare professionals, including clinical neurologists, physical medicine doctors, physiotherapists, speech therapists, and occupational therapists, use telerehabilitation extensively as part of a multidisciplinary team. Individuals sometimes could not reach this large multidisciplinary team quickly, resulting in inadequate patient recovery. In

\footnotetext{
*Correspondence: mehmet.ozkeskin76@gmail.com

${ }^{2}$ Department of Physiotherapy and Rehabilitation, Faculty of Health

Sciences, Ege University, İzmir, Turkey

Full list of author information is available at the end of the article
}

addition, supervised health care is often interrupted due to a lack of access to hospitals and clinics [2].

Telerehabilitation could be used to manage neurological diseases in both acute and chronic cases [3]. Telerehabilitation increases the effectiveness of treatment. On the other hand, remote rehabilitation services improve patient motivation, expectation, and satisfaction [4]. Telemedicine applications were assumed to provide an outstanding contribution to rehabilitation in terms of synchronous evaluation-treatment and training-consultancy services via video-conference methods [5].

Although telerehabilitation service was not found to be equivalent to one-to-one treatment, it would be effective in terms of cost-effectiveness, labor and time 
[6, 7]. Current telerehabilitation services are offered to patients through software, mobile applications, games, virtual reality, augmented reality, artificial intelligence, and video-conferencing or sensor-based devices $[8,9]$. Rehabilitation programs can become more efficient by increasing the motivation and participation of patients during the exercise [10].

To the best of our knowledge, exercise intervention studies via telerehabilitation have not been reviewed comprehensively. Demonstrating the purpose and effectiveness of telerehabilitation in neurological rehabilitation would significantly contribute to the literature. Emphasizing the parameters for which telerehabilitation is most efficient in terms of clinical practice may increase the effectiveness of treatment and rehabilitation. This study was aimed to present the current evidence of telerehabilitation in neurological rehabilitation patients.

\section{Materials and methods}

\section{Search strategy and selection criteria}

The search strategy of the study was carried out according to the recommendations of international guidelines [11]. One of the researchers was searched the literature via the PubMed database to find the studies. The physical exercise-based telerehabilitation studies within the scope of neurological rehabilitation were included, published between January 2015 and January 2021.

Keywords were determined as follows; "Multiple Sclerosis" and "Telerehabilitation" (n:58), "Stroke" and "Telerehabilitation" (n:190), "Parkinson's Disease" and "Telerehabilitation" (n:38), "Neuromuscular Disease" and "Telerehabilitation"(n:12), "Cerebellar Ataxia"” and "Telerehabilitation" (n:1), "Spinal Cord Injury" and "Telerehabilitation" (n:27), "Peripheric Nerve Injury" and "Telerehabilitation (n:0), "Polyneuropathies" and "Telerehabilitation" (n:0), "Intracranial Tumors" and "Telerehabilitation" (n:3).

A total of 329 studies were obtained during the initial search. Inclusion criteria of the study were; (1) the studies including a physical exercise program within the scope of the physiotherapy program and (2) having a randomized controlled design. Exclusion criteria of the study were; (1) studies for which only abstract available, (2) duplicate studies, and (3) studies including cases with traumatic brain injury. It was aimed to obtain a more homogeneous review by excluding studies that included traumatic brain injury cases. Because the sudden change in psychological status in individuals with traumatic brain injury may change the proportion of individuals who benefit from telemedicine.

\section{Evidence synthesis}

The researchers reached a consensus at the meeting on the inclusion of the articles. The qualitative/narrative synthesis was conducted. We also aimed to systematically present the studies with tables into categories [12].

\section{Results}

Inclusion and exclusion of the studies using Rayyan software yielded the following data: Multiple Sclerosis (MS) (3 studies), Stroke (6 studies), Parkinson's Disease (1 study), Intracranial Tumors (1 study), Spinal Cord Injury (1 study). In conclusion, a total of 12 randomized controlled studies were included in this narrative literature review (Fig. 1). The following information was collected: first author, year, intervention, evaluation parameters, and results (Tables 1, 2, 3).

\section{Discussion}

\section{Studies on multiple sclerosis}

Three of the 12 studies included in this review were conducted on individuals with MS. Considering that most MS patients had a relapsing-remitting type of disease, the importance of telerehabilitation is further understood [13]. Because urgent changes are required in the treatment program during MS attacks, two studies focused on balance and stabilization. In one of the studies, Conroy et al. investigated the effectiveness of a customized internet-based telerehabilitation on ambulation rate, balance, exercise compliance in MS patients. The intervention was included core stabilization and lower extremity functionality exercise training. The control group was received supervised exercise sessions. No difference was found in terms of physical performance, balance and walking in terms of three and six-month follow-up periods $(p>0.05)$ [14]. Since it is known that supervised exercises are effective in terms of balance and gait parameters in MS rehabilitation, it may be a different alternative telerehabilitation option to conduct balance programs via telemedicine in MS alternatively with sensory integration and other additional methods [15].

Muscle strength of MS patients significantly decreases due to fatigue and relatively diminished nerve conduction velocity during attack periods. Low muscle force reduces functionality and quality of life of the patients. Paul et al. evaluated telerehabilitation based exercise applications compared to a conventional home care program in MS patients. The authors recommended physical exercise (two days a week) to their patients for 26 weeks. As part of an individualized exercise program through web-based physiotherapy, patients were given cardiovascular, strength and balance exercises as well as warming, cooling and stretching exercises. The conventional 

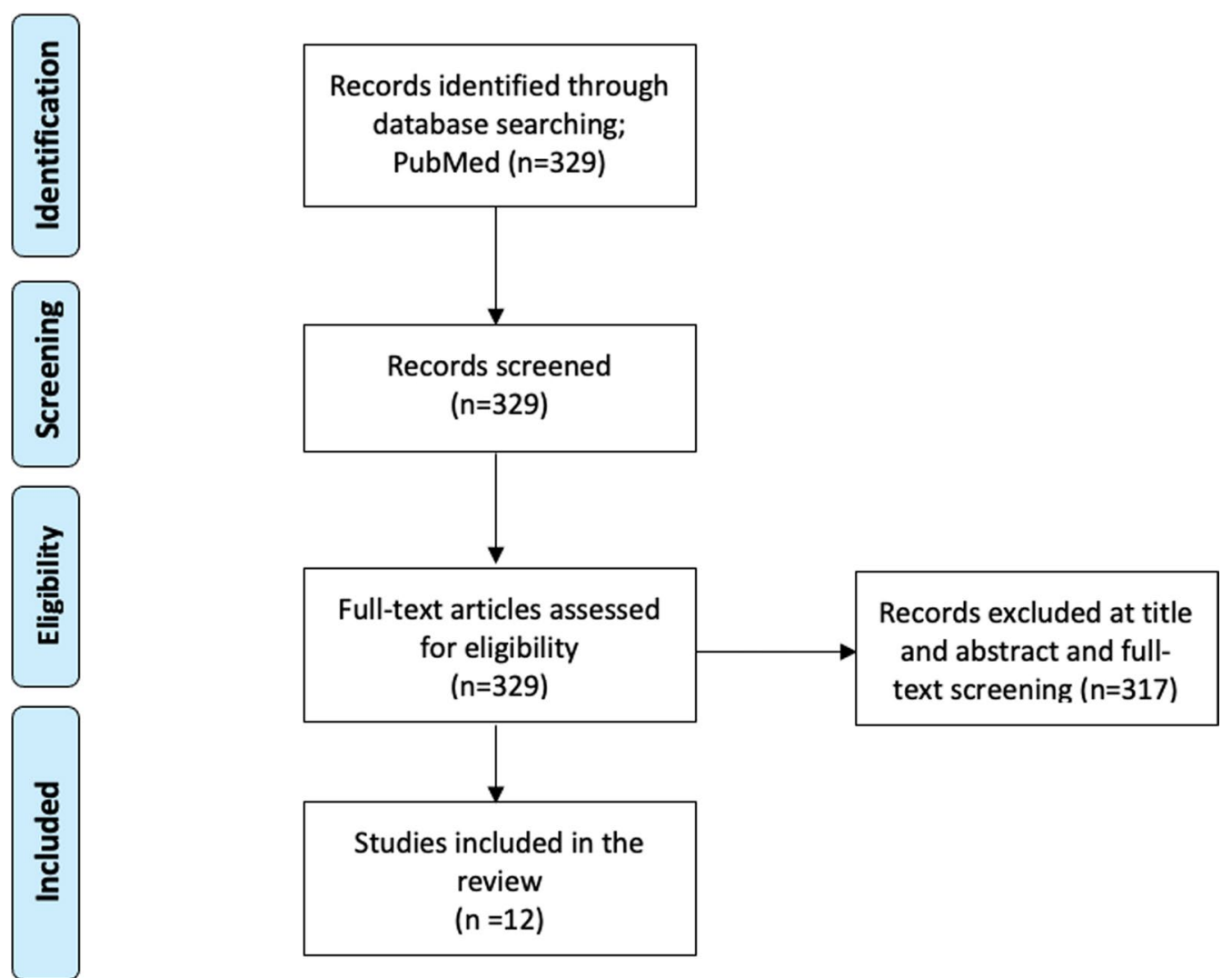

Fig. 1 CONSORT diagram of the study

group received the same exercise prescription with a paper guide. There was no significant difference between the web-based physiotherapy and standard home exercise groups in any parameter at the 3rd-month follow-up $(p>0.05)$. A significant difference was found between the web-based physiotherapy and standard home exercise groups in favor of the web-based exercise group only in the EQ-5D-VAS parameter at the 6th-month evaluations $(p<0.05)[16]$. These results suggested that telerehabilitation could improve the overall quality of life in MS patients. However, there is a need for more comprehensive research on whether this situation occurs with a direct effect on clinical parameters or through the satisfaction and motivation parameters brought by better compliance with the clinician [17].

In another study with the 24-week exercise program to evaluate the effect of web-based protocol training on quality of life in MS patients, training support with was presented asynchronously via a message, e-mail and telephone. Exercise band and medicine balls were used as additional material in the training sessions. Exercise intensity was adjusted with the Borg Scale. Strength and endurance exercises was performed once a week. On the other hand, the control group continued their previous physical activities and were followed up for 3 months. There was no significant difference between the two MS groups regarding health-related quality of life and fatigue $(p>0.05)$. In the intervention group, knee extension and knee flexion motion were significantly higher at three months $(p<0.05)$. Both knee extension, knee flexion, trunk flexion and extension were significantly higher in favor of the intervention group at the 6th month $(p<0.05)$. There was a significant difference in the intervention group compared to the control group after only three months of training $(p<0.05)$ [18]. Improvements were reported in strength and endurance. However, psychological parameters, including fatigue and quality of life, did not positively affected. We assumed that more subjective data was required to point out the effectiveness of telehealth on the psychological state of neurology patients.

\section{Studies on stroke}

Stroke was the most frequent case group in our study to yield telerehabilitation. Because the transfer of stroke patients to the hospital is difficult. It is essential to manage both home care and other health services remotely in some cases. Since stroke requires life-long rehabilitation, 


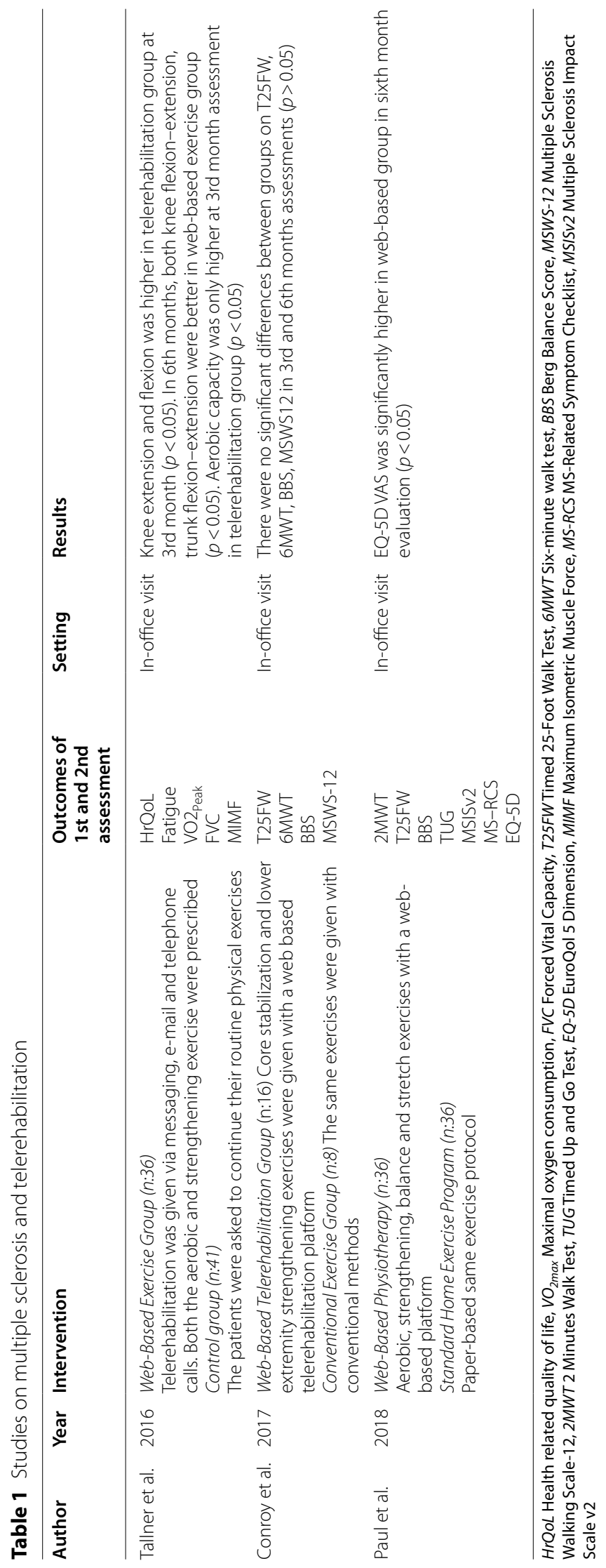


Table 2 Studies on stroke and telerehabilitation

\begin{tabular}{|c|c|c|c|c|c|}
\hline Author & Year & Intervention & $\begin{array}{l}\text { Outcomes of } \\
1 \text { st and } 2 \text { nd } \\
\text { assessment }\end{array}$ & Setting & Results \\
\hline Linder et al. & 2015 & $\begin{array}{l}\text { Telerehabilitasyon group ( } n: 51 \text { ) } \\
\text { Hand Mentor Pro robotic assisted wrist and } \\
\text { finger active assistive range of motion } \\
\text { Home exercise group ( } n: 48) \\
\text { Active, active-assisted, and functional } \\
\text { range of motion exercises }\end{array}$ & $\begin{array}{l}\text { SIS } \\
\text { CES-D }\end{array}$ & In-office visit & $\begin{array}{l}\text { There was significant difference between } \\
\text { groups on hand function }(p<0.05)\end{array}$ \\
\hline Chumbler et al. & 2015 & $\begin{array}{l}\text { Telerehabilitasyon group (STeleR) (n:25) } \\
\text { Consisted of telephone and message- } \\
\text { based function exercises and adaptive } \\
\text { techniques for the daily life } \\
\text { Conventional Care ( } n: 23 \text { ) } \\
\text { Conventional rehabilitation care was } \\
\text { applied to this group }\end{array}$ & $\begin{array}{l}\text { FES } \\
\text { SSPS-CS }\end{array}$ & Remotely & $\begin{array}{l}\text { STeleR group was better on satisfaction } \\
(p<0.05) \text {. There were no significant differ- } \\
\text { ences on FES }(p>0.05)\end{array}$ \\
\hline Chen et al. & 2017 & $\begin{array}{l}\text { Telerehabilitasyon group (n:26) } \\
\text { Live video-conferencing was conducted by } \\
\text { physiotherapist } \\
\text { Conventional Physiotherapy Group(n:25) } \\
\text { Conventional face-to-face physiotherapy } \\
\text { was applied }\end{array}$ & $\begin{array}{l}\mathrm{mBI} \\
\mathrm{BBS}\end{array}$ & In-office visit & $\begin{array}{l}\text { There was no significant difference between } \\
\text { groups }(p>0.05)\end{array}$ \\
\hline Chen et al. & 2020 & $\begin{array}{l}\text { Home-Based Telerehabilitasyon ( } n: 22 \text { ) } \\
\text { Telerehabilitation based home exercise } \\
\text { program was applied with the assistance of } \\
\text { physiotherapist } \\
\text { Conventional Physiotherapy (n:22) } \\
\text { Consists of face-to-face physiotherapy } \\
\text { sessions }\end{array}$ & $\begin{array}{l}\text { FMA } \\
\mathrm{BI}\end{array}$ & In-office visit & $\begin{array}{l}\text { Telerehabilitation group was better in terms } \\
\text { of hand function }(p<0.05)\end{array}$ \\
\hline Wu et al. & 2020 & $\begin{array}{l}\text { Intervention Group (n:32) } \\
\text { Multi-disciplinary care model was applied } \\
\text { via the teleconferencing } \\
\text { Control Group (n:32) } \\
\text { Extremity position, transfer activities, range } \\
\text { of motion exercises was prescribed }\end{array}$ & $\begin{array}{l}\text { FMA } \\
\text { BBS } \\
6 M W T \\
\text { mBI } \\
\text { SSQoL }\end{array}$ & In-office visit & $\begin{array}{l}\text { Telerehabilitation group was significantly } \\
\text { higher score on Fugl-Meyer Motor Function } \\
\text { Assessment, Berg Balance Scale ve Stroke- } \\
\text { Specific Quality of Life Scale }(p<0.05)\end{array}$ \\
\hline Asano et al. & 2021 & $\begin{array}{l}\text { Telerehabilitation-Based Physiotherapy (n:50) } \\
\text { Standardized telerehabilitation, includ- } \\
\text { ing physiotherapy and ergotherapy was } \\
\text { carried out } \\
\text { Conventional Care Group (n:48) Standard } \\
\text { hospital care was conducted }\end{array}$ & $\begin{array}{l}\text { LLFDI } \\
\text { SF-36 } \\
\text { TFMWT } \\
\text { 2MWT } \\
\text { mBI }\end{array}$ & In-office visit & $\begin{array}{l}\text { There was no significant difference between } \\
\text { groups }(p>0.05)\end{array}$ \\
\hline
\end{tabular}

SIS Stroke Impact Scale, CES-D Center for Epidemiologic Studies Depression Scale, FES Falls Efficacy Scale, T25FWTimed 25-Foot Walk Test, $m B I$ Modified Barthel Index, BBS Berg Balance Score, FMA Fugl-Meyer Assessment, BI Barthel Index, 6MWT Six-minute walk test, SF-36 Short Form 36, TFMWT Timed Five-Meter Walk Test, $2 M W T$ 2 Minutes Walk Test, SSPS-CS Stroke-Specific Patient Satisfaction with Care Scale, SSQoL Stroke Specific Quality of Life Scale, LLFDI Late-Life Function and Disability Instrument

the accessibility of telerehabilitation provides essential contribution to the patients [19]. Linder et al. planned a randomized controlled trial to determine the effectiveness of remote home exercise program (HEP) on quality of life and depression in people after stroke. The exercise program included range of motion exercises: active, active-assisted movements, and functional exercises with the Hand Mentor Pro robot-assisted device for eight weeks ( 5 days/week, $3 \mathrm{~h}$ a day). There was no significant difference between the two groups regarding function and depression $(p>0.05)$ [20]. In particular, the ineffectiveness of a telerehabilitation service including robotics in stroke, may be due to the system's complexity.
Additionally, the 8-week follow-up period may not be enough time to observe this possible positive effect.

Chumbler et al. determined the impact of multifactorial stroke telerehabilitation on satisfaction in patients with stroke, who had fall history. Multifactorial Stroke Telerehabilitation Group received telerehabilitation via messaging, telephone calls to give patients function exercises and adaptive daily life techniques. Conventional care was applied to the control group. Compared with the control group, intervention group showed significant changes in hospital satisfaction $(p<0.05)$ [21]. No difference was observed in self-efficacy due to falling. It is known that telerehabilitation does not affect clinical parameters in 
Table 3 Studies on other neurologic disorders and telerehabilitation

\begin{tabular}{|c|c|c|c|c|c|c|}
\hline Author & Year & Disorder & Intervention & $\begin{array}{l}\text { Outcomes of } \\
1 \text { st and } 2 \text { nd } \\
\text { assessment }\end{array}$ & Setting & Results \\
\hline Gandolfi et al. & 2017 & Parkinson's Disease & $\begin{array}{l}\text { TeleWii Group ( } n: 38 \text { ) } \\
\text { Home-based TeleWii education } \\
\text { was consisted of } 21 \text { balance exer- } \\
\text { cise session, each of } 50 \text { min } \\
\text { Sensory Integration Balance Edu- } \\
\text { cation ( } n: 34 \text { ) } \\
\text { Static, dynamic balance and gait } \\
\text { education was consisted of } 21 \\
\text { balance exercise session, each of } \\
50 \text { min }\end{array}$ & $\begin{array}{l}\text { BBS } \\
\text { ABC } \\
10 M W T \\
\text { DGI }\end{array}$ & In-office visit & $\begin{array}{l}\text { TeleWii group was higher in terms } \\
\text { of balance }(p<0.05)\end{array}$ \\
\hline Coulter et al. & 2017 & Spinal Cord Injury & $\begin{array}{l}\text { Web-Based Physiotherapy Group } \\
\text { (n:15) } \\
\text { Video-based exercise, exercise } \\
\text { diary was ordered for } 8 \text { weeks } \\
\text { Conventional Care Group (n:6) } \\
\text { Routine physical activity was } \\
\text { encouraged and patients were } \\
\text { asked to fill exercise diary }\end{array}$ & $\begin{array}{l}6 \mathrm{MWT} \\
\text { WHOQOL } \\
\text { HADS }\end{array}$ & In-office visit & $\begin{array}{l}\text { There was no significant difference } \\
\text { between groups }(p>0.05)\end{array}$ \\
\hline Gehring et al. & 2018 & Intra-cranial Tumor & $\begin{array}{l}\text { Home Telerehabilitation Group } \\
\text { (n:23) } \\
\text { Maximum heart rate was set as } \\
\% 60-85 . \text { Aerobic exercises were } \\
\text { applied and monitorized } \\
\text { Control Group (n:11) } \\
\text { Active life-style prescriptions } \\
\text { were ordered regarding national } \\
\text { health guidelines }\end{array}$ & $\begin{array}{l}I P A Q \\
\mathrm{VO}_{2 \max } \\
\mathrm{BMI}\end{array}$ & In-office visit & $\begin{array}{l}\text { Telerehabilitation group was better } \\
\text { on } \mathrm{VO}_{2} \text { peak and } \mathrm{BMI}(p<0.05)\end{array}$ \\
\hline
\end{tabular}

BBS Berg Balance Score, ABC Activity Specific Balance Confidence, 10MWT 10-Meter Walking Test, DGI Dynamic Gait Index, 6MWT Six-minute walk test, WHOQoL World Health Organization Quality of Life assessment, HADS Hospital Anxiety Depression Scale, IPAQ International Physical Activity Questionnaire, VO ${ }_{2 m a x}$ Maximal oxygen consumption, BMI Body Mass Index

some cases. On the other hand, it has a positive effect in the long term by increasing satisfaction and participation [22]. It can be argued that this psychological effect may also contribute to the physical condition over the years.

In another study, Chen et al. were aimed to provide the effectiveness of a 12-week telerehabilitation program of motor exercises in stroke patients. One hour of physiotherapy and 10 rehabilitation training sessions per week were applied in a 3-month program. Under the guidance of physiotherapists, the telerehabilitation system and the face-to-face training program were compared with inhome rehabilitation training. The telerehabilitation group showed a significant improvement in upper extremity performance compared to the conventional rehabilitation group $(p<0.05)$ [23]. These results may suggest that telerehabilitation is more effective than face-to-face training in remote stroke management. We also believe that patients may have worked more efficiently in their home setting.

To investigate the effectiveness of exercise training based telerehabilitation on patients with acute stroke, Wu et al. designed a comprehensive study for home care. The training was given to the patients, including extremity positioning, bed positioning, transfer activities and joint mobility for three months. After the patients were discharged, routine rehabilitation recommendations were applied to the patients in the control group via phone follow-up once a week. Significant changes were observed in both intervention and control groups compared to baseline $(p<0.05)$. However, intervention group gained more on "Fugl-Meyer Motor Function Assessment", "Berg Balance Scale" and "Stroke-Specific Quality of Life Scale" $(p<0.05)$ [24]. These results suggest that telerehabilitation can be an effective care model in the acute period [25]. In order to improve balance, hand function and quality of life of stroke patients in the acute period, the remote care model may benefit clinical practice.

Chen et al. conducted a 3-month program to assess the effectiveness of home telerehabilitation on physical performance in stroke patients. After discharge, both groups were given exercises ("Bobath Therapy" and "Proprioceptive Neuromuscular Facilitation") and ElectromyographyTriggered Neuromuscular Stimulation". The conventional physiotherapy group received rehabilitation treatment in the outpatient physical therapy unit. There were significant improvements in function and balance parameters 
in both groups $(p<0.05)$, but none of the differences between the groups was significant $(p>0.05)$ [26]. Due to the complex nature of the "Bobath Therapy" and "Proprioceptive Neuromuscular Facilitation" exercises applied in this study, better results could not be obtained compared to the conventional rehabilitation group. Further studies may prefer simple exercises within the scope of remote rehabilitation. Comparing complex and simple exercise prescriptions in randomized controlled trial may further explain this situation.

Asano et al. planned a study to assess the effect of the telerehabilitation system on functional results after the 3 months of stroke. The telerehabilitation group received both an occupational therapy and a physiotherapy program. The study physiotherapist revised the exercises with a personalized program. The usual care group received the routine care (1-h clinic-based rehabilitation sessions once or twice a week) until to the discharge. No significant difference was found in any parameter between the groups $(p>0.05)$ [27]. It may also be the case that the clinical picture affects the efficiency of telerehabilitation in individuals with stroke. Remote rehabilitation may be less effective, especially in the very old-aged and disabled population, also depending on the sociocultural level.

\section{Studies on other neurological disorders}

Another case group was the Parkinson's disease. Gandolfi et al. was planned research to demonstrate the effect of in-home virtual reality based telerehabilitation on balance. The control group was received "Sensory Integration-Based Balance Training (SIBT)" in the face-to-face application. Both groups received 21 individualized treatment sessions of $50 \mathrm{~min}$. The frequency of the exercises was 3 days for week. Static and dynamic balance training were given with a SIBT approach. Balance was found to be significantly higher in virtual reality group $(p<0.05)$. There was no significant difference in other outcome measures $(p>0.05)$ [28]. Virtual Reality can give effective results in balance training. On the other hand, it should be considered that the sustainability of the balance results on the function is controversial.

The other case group was intra-cranial tumors. Gehringer et al. evaluated the effect of a home-based exercise for patients with high-level glioma. The program lasted a total of 24 weeks. The home-based group was given an aerobic exercise prescription with a maximal heart rate adjusted to $60-85 \%$. Compared with the control group, the intervention group presented significant improvements in absolute $\mathrm{VO}_{2 \max }$ and body mass index $(p<0.05)$ [29]. It should be emphasized that remote aerobic exercise follow-up and monitoring can also be effective in neurological rehabilitation [30].
It is also known that the efficacy of telerehabilitation in the management of spinal cord injuries has already been proven. Coulter et al. planned an 8-week, 2 sessions per week exercise program to assess the effect of webbased tele-exercise protocol in participants with spinal cord injury. The web-based physiotherapy group used the website for personalized exercise programs. Each exercise page includes a video and a written description of the exercise. 30-min sessions were given 2 days a week for 8 weeks. Ordinary care group was received conventional exercise program. Although the differences between the groups were not significant, six-meter walk test performance was higher in web-based physiotherapy group $(p>0.05)$. No significant differences were found between the groups for other parameters $(p>0.05)$ [31]. We assumed that exercise progression via video conference would provide additional contributions to the webbased applications.

\section{Conclusions}

Most of the telerehabilitation studies were conducted in the stroke cases. Telerehabilitation was found to be effective on patient satisfaction as well as clinical parameters including hand function, balance and function in patients with stroke. On the other hand, it was observed that telerehabilitation positively affected the general quality of life, muscle strength and endurance in individuals with MS. Balance and function improved with telerehabilitation in individuals with Parkinson's disease. Lastly, aerobic capacity increased in individuals with intra-cranial tumor and spinal cord injury via telehealth applications. It has been emphasized that the most significant barriers to access to telerehabilitation are the advanced age of the patients and the problems of accessing the internet. In summary, physical exercise with telerehabilitation provides productive results to improve quality of life, muscle strength-endurance, hand function, balance, aerobic capacity in patients recruiting neurologic rehabilitation.

\footnotetext{
Abbreviations

MS: Multiple sclerosis; MeSH: Medical Subject Headings; EQ-5D-VAS: EuroQol-5D-Visual Analog Scale; HEP: Home Exercise Program; STeleR: Stroke Telerehabilitation Group; PNF: Proprioceptive neuromuscular facilitation; ETNS: Electromyography-triggered neuromuscular stimulation; SIBT: Sensory integration based balance training; 6MWT: Six Meter Walk Test; T25FW: Timed 25-FootWalk Test (T25FW); BBS: Berg Balance Score; MSWS-12: MS Walking Scale-12; 2MWT: 2 Minute-Walk Test (2MWT); TUG: Timed up and go test; HrQoL: Health Related Quality of Life; FVC: Forced vital capacity; SIS: Stroke Impact Scale; CES-D: Center for Epidemiologic Studies Depression Scale; FES: Falls Efficacy Scale; SSPSC: Stroke-Specific Patient Satisfaction with Care; FMA Fugl-Meyer Assessment; mBI: Modified Barthel Index; SF-36: Short Form 36 (SF-36); TFMWT:Timed Five-Meter Walk Test; ABC: Activities-Specific Balance Confidence; 10MWT: 10-Meter Walking Test; DGI: Dynamic Gait Index; IPAQ: International Physical Activity Questionnaire (IPAQ); BMI: Body Mass Index; HADS: Hospital Anxiety Depression.
} 


\section{Acknowledgements \\ Not applicable.}

\section{Authors' contributions}

SMA and MÖ researched literature and conceived the study. FÖ and MÖ were involved in protocol development and writing. All authors reviewed and edited the manuscript and approved the final version of the manuscript.

\section{Funding}

None.

Availability of data and materials

Not applicable.

\section{Declarations}

Ethics approval and consent to participate

Not applicable.

\section{Consent for publication}

Not applicable.

\section{Competing interests}

The authors declare that they have no competing interests.

\section{Author details}

${ }^{1}$ Department of Health Care Services, Köyceğiz Vocational School of Health Services, Muğla Sıtkı Koçman University, Muğla, Turkey. ${ }^{2}$ Department of Physiotherapy and Rehabilitation, Faculty of Health Sciences, Ege University, Izmir, Turkey. ${ }^{3}$ Department of Physiotherapy and Rehabilitation, Institute of Health Sciences, Ege University, İzmir, Turkey.

Received: 3 November 2021 Accepted: 7 February 2022

Published online: 19 February 2022

\section{References}

1. Schwamm LH, Holloway RG, Amarenco P, Audebert HJ, Bakas T, Chumbler NR, et al. A review of the evidence for the use of telemedicine within stroke systems of care: a scientific statement from the American Heart Association/American Stroke Association. Stroke. 2009;40(7):2616-34.

2. Koh GC, Yen SC, Tay A, Cheong A, Ng YS, De Silva DA, et al. Singapore Tele-technology Aided Rehabilitation in Stroke (STARS) trial: protocol of a randomized clinical trial on tele-rehabilitation for stroke patients. BMC Neurol. 2015;15(1):1-4.

3. Wootton R. Twenty years of telemedicine in chronic disease management-an evidence synthesis. J Telemed Telecare. 2012;18(4):211-20.

4. Medina JL, Acosta-Vargas P, Rybarczyk Y. A systematic review of usability and accessibility in telerehabilitation systems. London: IntechOpen; 2019.

5. Cardinale AM. The opportunity for telehealth to support neurological health care. Telemed E-Health. 2018;24(12):969-78.

6. Bendixen RM, Levy CE, Olive ES, Kobb RF, Mann WC. Cost effectiveness of a telerehabilitation program to support chronically ill and disabled elders in their homes. Telemed E-Health. 2009:15(1):31-8.

7. Fatoye F, Gebrye T, Fatoye C, Mbada CE, Olaoye MI, Odole AC, et al. The clinical and cost-effectiveness of telerehabilitation for people with nonspecific chronic low back pain: randomized controlled trial. JMIR Mhealth Uhealth. 2020;8(6):e15375

8. Calvaresi D, Marinoni M, Dragoni AF, Hilfiker R, Schumacher M. Realtime multi-agent systems for telerehabilitation scenarios. Artif Intell Med. 2019:96:217-31

9. de Souza Miguel GF, de Sá AAR, de Souza JT, Naves ELM. Home-based telerehabilitation: a review of remote therapy frameworks. Res Soc Dev. 2021;10(6):e4910615489.

10. Mella-Abarca W, Barraza-Sánchez V, Ramírez-Parada K. Telerehabilitation for people with breast cancer through the COVID-19 pandemic in Chile. Ecancermedicalscience. 2020;14:1-8.
11. Ferrari R. Writing narrative style literature reviews. Med Writ. 2015;24(4):230-5

12. Popay J, Roberts $H$, Sowden A, Petticrew M, Arai L, Rodgers M, et al. Guidance on the conduct of narrative synthesis in systematic reviews: a product from the ESRC Methods Programme. Lancaster: Lancaster University; 2006.

13. Adelman G, Rane SG, Villa KF. The cost burden of multiple sclerosis in the United States: a systematic review of the literature. J Med Econ. 2013;16(5):639-47.

14. Conroy SS, Zhan M, Culpepper WJ, Royal W III, Wallin MT. Self-directed exercise in multiple sclerosis: evaluation of a home automated telemanagement system. J Telemed Telecare. 2018;24(6):410-9.

15. Gandolfi M, Munari D, Geroin C, Gajofatto A, Benedetti MD, Midiri A, et al. Sensory integration balance training in patients with multiple sclerosis: a randomized, controlled trial. Mult Scler. 2015:21(11):1453-62.

16. Paul L, Renfrew L, Freeman J, Murray H, Weller B, Mattison P, et al. Web-based physiotherapy for people affected by multiple sclerosis: a single blind, randomized controlled feasibility study. Clin Rehabil. 2019:33(3):473-84.

17. Özden F, Sarı Z, Karaman ÖN, Aydoğmuş H. The effect of video exercise-based telerehabilitation on clinical outcomes, expectation, satisfaction, and motivation in patients with chronic low back pain. Ir J Med Sci (1971-). 2021;1:1-11.

18. Tallner A, Streber R, Hentschke C, Morgott M, Geidl W, Mäurer M, et al. Internet-supported physical exercise training for persons with multiple sclerosis - a randomised, controlled study. Int J Mol Sci. 2016;17(10):1-11.

19. Johansson T, Wild C. Telerehabilitation in stroke care-a systematic review. J Telemed Telecare. 2011;17(1):1-6.

20. Linder SM, Rosenfeldt AB, Bay RC, Sahu K, Wolf SL, Alberts JL. Improving quality of life and depression after stroke through telerehabilitation. Am J Occup Ther. 2015;69(2):1-10.

21. Chumbler NR, Li X, Quigley P, Morey MC, Rose D, Griffiths P, et al. A randomized controlled trial on Stroke telerehabilitation: The effects on falls self-efficacy and satisfaction with care. J Telemed Telecare. 2015;21(3):139-43.

22. Piron L, Turolla A, Tonin P, Piccione F, Lain L, Dam M. Satisfaction with care in post-stroke patients undergoing a telerehabilitation programme at home. J Telemed Telecare. 2008;14(5):257-60.

23. Chen J, Sun D, Zhang S, Shi Y, Qiao F, Zhou Y, et al. Effects of homebased telerehabilitation in patients with stroke: a randomized controlled trial. Neurology. 2020;95(17):e2318-30.

24. Wu Z, Xu J, Yue C, Li Y, Liang Y. Collaborative care model based telerehabilitation exercise training program for acute stroke patients in China: a randomized controlled trial. J Stroke Cerebrovasc Dis. 2020:29(12):105328.

25. Corriveau H, Tousignant M, Gosselin S, Boissy P. Patients satisfaction with an in-home telerehabilitation exercise program and physiotherapists' satisfaction toward technology for an acute stroke population: a pilot study. Assistive technology: from research to practice. Amsterdam: IOS Press; 2013. p. 753-7.

26. Chen J, Jin W, Dong WS, Jin Y, Qiao FL, Zhou YF, et al. Effects of homebased telesupervising rehabilitation on physical function for stroke survivors with hemiplegia: a randomized controlled trial. Am J Phys Med Rehabil. 2017;96(3):152-60.

27. Asano M, Tai BC, Yeo FY, Yen SC, Tay A, Ng YS, et al. Home-based telerehabilitation presents comparable positive impact on self-reported functional outcomes as usual care: the Singapore Tele-technology Aided Rehabilitation in Stroke (STARS) randomised controlled trial. J Telemed Telecare. 2021;27(4):231-8.

28. Gandolfi M, Geroin C, Dimitrova E, Boldrini P, Waldner A, Bonadiman S, et al. Virtual reality telerehabilitation for postural instability in Parkinson's disease: a multicenter, single-blind, randomized, controlled trial. Biomed Res Int. 2017;2017:7962826-911.

29. Gehring K, Kloek CJ, Aaronson NK, Janssen KW, Jones LW, Sitskoorn MM, et al. Feasibility of a home-based exercise intervention with remote guidance for patients with stable grade II and III gliomas: a pilot randomized controlled trial. Clin Rehabil. 2018;32(3):352-66.

30. Aragaki D, Luo J, Weiner E, Zhang G, Darvish B. Cardiopulmonary telerehabilitation. Phys Med Rehabil Clin. 2021:32(2):263-76. 
31. Coulter EH, McLean AN, Hasler JP, Allan DB, McFadyen A, Paul L. The effectiveness and satisfaction of web-based physiotherapy in people with spinal cord injury: a pilot randomised controlled trial. Spinal Cord. 2017:55(4):383-9.

\section{Publisher's Note}

Springer Nature remains neutral with regard to jurisdictional claims in published maps and institutional affiliations.

\section{Submit your manuscript to a SpringerOpen ${ }^{\circ}$ journal and benefit from:}

- Convenient online submission

- Rigorous peer review

- Open access: articles freely available online

- High visibility within the field

- Retaining the copyright to your article

Submit your next manuscript at $\boldsymbol{\nabla}$ springeropen.com 